\title{
Reflexiones de un diplomático. ¿Puede la política exterior ser un instrumento que contribuya a mejorar la competitividad y la calidad institucional de la República Argentina?
}

\author{
Carlos Sersale Di Cerisano*
}

\section{Resumen}

Si se quisiera sintetizar la problemática argentina en dos causas principales, las cuales cruzan absolutamente todas las áreas que limitan su crecimiento económico sostenido y desarrollo sustentable, éstas podrían sintetizarse en su competitividad y en su falta de transparencia institucional.

En este contexto, y partiendo del supuesto que los cambios estructurales tienen que provenir de la agenda interna del país, se intenta en este artículo de opinión, señalar cómo la política exterior y sus alianzas internacionales relacionadas pueden ser un instrumento para controlar esas causas y contribuir a la construcción de un estado inclusivo, capaz de producir esos cambios que limitan su crecimiento económico y desarrollo sustentable.

Un elemento clave para que la política exterior sea eficaz y eficiente es el capital humano. Es así que la membrecía al Servicio Exterior de la Nación debe combinar los valores esenciales requeridos al diplomático y a su comprensión de la real problemática argentina, con el ejercicio de sus actividades en el país y en el exterior, vinculando la teoría y la práctica.

Cuando se hace referencia al diseño de una estrategia de política exterior y de sus instrumentos, se parte del principio: "la política exterior comienza por casa" (Haass, 2013) y, para que ella sea eficaz se debe contar con un estado que sea capaz de gestionar esa estrategia de política y sus instrumentos, ya sea interna como internacionalmente, no solamente a nivel del estado (incluyendo los "títulos" y "secciones" establecidos por la Constitución Nacional), sino también con la sociedad civil en absolutamente todas sus áreas específicas.

Ahora bien, ello no puede realizarse sin el capital humano para su implementación. No se trata solamente de su profesionalización, la cual es - ciertamente- un condicionante clave para garantizar continuidad en la aplicación de las estrategias y políticas decididas a nivel del gobierno federal, sino además de los aspectos cualitativos del "cómo" desarrollar ese capital humano. Para ello, el diplomático debe tener incorporado ciertos "valores centrales" no sólo en su forma de ser, sino también en su formación profesional y en sus actividades. Representar al país en el exterior es una misión de $24 \mathrm{hs}$ sobre $24 \mathrm{hs}$.

El diplomático (incluyendo sus funciones consulares) debe enfocar su trabajo con integridad; contar con vocación de servidor público; debe tener capacidad de identificar las oportunidades que brinda la agenda internacional para generar las políticas multilaterales y bilaterales que contribuyan a mejorar la calidad de vida del pueblo argentino y de sus instituciones; está obligado a identificar las "buenas prácticas" de terceros países y organizaciones internacionales que estime van a ser útiles para superar los cuellos de botella que afectan -en todas sus dimensiones- a la Argentina. Debe tener en cuenta que las relaciones internacionales hoy ya no se caracterizan sólo por la relación Estado-Estado o Estado-

* Licenciado en Ciencias Economicas y Dr en Ciencia Política. Embajador de carrera de la República Argentina (r). Subdirector del Comité de Política Exterior y FFAA del CARI. E-mail: carlossersale@yahoo.com.ar 
organismo internacional y que, por lo tanto sus funciones incluyen su relacionamiento con la sociedad civil en todas sus áreas específicas. Por ello debe demostrar su compromiso y capacidad de ejecución con las políticas, programas y proyectos según el puesto que ocupe y obviamente tener una buena relación con sus colegas y empleados. Finalmente, tener la capacidad de trabajar efectivamente con individuos de todas las culturas, además de mantener a lo largo de toda su vida laboral un compromiso personal hacia el aprendizaje y hacia su desarrollo profesional.

\section{¿Cómo se traducen estos valores arriba mencionados en capacidades concretas?}

a) liderazgo para implementar la visión de país que se pretende representar y generar oportunidades para la Argentina en absolutamente todas las áreas posibles (siempre pensando en términos de beneficio mutuo con la contraparte que se trate, ya sea pública o sociedad civil)

b) enfocar el tema con una perspectiva estratégica y contar con la influencia necesaria para generar las acciones relacionadas.

c) desarrollar la capacidad para manejar situaciones complejas en términos de coordinación, organización, flexibilidad en materia de capacidad de decisión y sobre todo, juicio/racionalidad analítica.

d) manejar las relaciones interpersonales a través del compromiso para trabajar en equipo, con capacidad de comunicación y manejo del conflicto y de situaciones estresantes.

Ahora bien, ¿por qué ser tan específico en el desarrollo de estas capacidades? Cierto es que debieran ser componentes de todos quienes son "operadores" de la política exterior de un país. No obstante, en el caso de la Argentina, su Cuerpo Permanente debe tener en claro que los valores y capacidades arriba desarrolladas deben ser más visibles que en los casos de los países que cuentan con instituciones sólidas, con capacidad de "hard power" (en todas sus dimensiones, públicas y privadas) y con un peso-país que hacen relativamente más fácil la labor de sus representantes diplomáticos, ya sea en el exterior como en sus funciones en el país.

Los diplomáticos argentinos, por el contrario, deben agudizar su capacidad de promover las múltiples características argentinas vinculadas al "soft power", incluyendo sus condiciones personales como diplomáticos y su análisis de fondo de las áreas de complementariedad posibles con el país u organismo internacional ante el cual se encuentran acreditados o frente al interlocutor que tengan ante sí mientras tengan funciones en la sede central.

Ello es así por los siguientes factores: por representar un país de desarrollo intermedio (y con una tendencia decreciente desde hace décadas); por su cada vez menor capacidad relativa de influir en los procesos de decisión globales; por la tendencia -en general- de "la política" a subestimar el rol de la política exterior con la idea que los errores no tienen costos haciendo una analogía- en muchos casos- con la política interna, errores que tardan años en subsanarse y afectan a la credibilidad del país y a su seguridad jurídica. A su vez, y lo más difícil, que las acciones de complementariedad con los países u organismos internacionales ante los cuales se encuentran acreditados no sea el resultado de "impulsos" basados en su creatividad y compromiso con el país, sino que sean el corolario de gestiones que se prolonguen en el largo plazo.

El discurso acerca del "potencial argentino" ya no es suficiente si no se lo justifica desde la racionalidad, desde el beneficio mutuo con la contraparte, desde las eventuales competitividades sectoriales, desde la transparencia institucional. Esto significa mostrar un país donde prevalezca la seguridad jurídica, el respeto al derecho a la propiedad y sobre todo evitar la tendencia sobre-reguladora (en los tres niveles del ejecutivo), donde se duplican y superponen normas que afectan negativamente la vida, no sólo de los argentinos sino de eventuales interesados en generar actividades conjuntas. 


\section{Ahora bien, ¿cómo se traduce este diagnóstico, recomendaciones y directrices institucionales al actual contexto?}

La crisis global provocada por el COVID 19 ha tenido, tiene y tendrá por un largo periodo diferentes impactos en absolutamente toda la vida de las naciones y de sus sociedades. En el caso argentino, ha puesto de manifiesto cuestiones que están directamente relacionadas con la estructura del Estado, con su rol, con sus funciones y con su capacidad de gestión. Ello ha afectado a todas las áreas en las cuales está directamente involucrado, particularmente en la social (en todas sus dimensiones, no solamente en su sistema de salud) y en la económica, ya sea pública o privada. La capacidad de respuesta del Estado no puede ser evaluada dejando de lado su actual estructura y su capacidad de gestión, las cuales no han dado los resultados óptimos a las diferentes situaciones que la profundización de esta crisis constante (y hoy sanitaria) ha provocado.

El rol del estado, su capacidad de gestión, sus vínculos con el sector privado en particular y con la sociedad civil en general, son un área donde las buenas prácticas de terceros países, sus experiencias y las alianzas estratégicas internacionales cuentan. Es obligación de los miembros del cuerpo diplomático en el exterior de identificar las oportunidades de cooperación bilateral y multilateral en todas las áreas y en contribuir -cada uno según sus funciones- para paliar los efectos de las crisis. Los funcionarios destinados en la sede central, en colaboración con las restantes áreas del estado y de la sociedad civil deben adaptarlas a las necesidades locales, teniendo en cuenta su cultura, costumbres y códigos.

Si conectamos esta crisis coyuntural, -agudizada por factores estructurales y que se prolongará en el tiempo- con diversos "síntomas", veremos que son cruciales los conceptos de "competitividad" y falta de "transparencia institucional" que cruzan todas las aéreas del estado, muchas del sector privado, incluyendo el funcionamiento de tantas organizaciones de la sociedad civil. Son cruciales, no sólo para transformar esta realidad que nos atrasa cada día más, sino también para elaborar una estrategia de política exterior que permita superar esas limitaciones a través de la cooperación internacional y de la adopción y adaptación de las buenas prácticas que han tenido éxito en terceros países.

Ambos conceptos no son coyunturales. Los cuellos de botella que generan estos factores se arrastran desde hace décadas y, en algunos casos desde que la Argentina se constituyó como Nación (Sersale di Cersiano, 2002).

Las problemáticas que hacen a la competitividad y a la falta de transparencia institucional incluyen la mayoría de los factores y variables que hacen al atraso, a la falta de modernización de sus instituciones, a su estructura económica y social y que han contribuido desde hace décadas a la decadencia argentina y a su condición de país en "subdesarrollo creciente". El rol del Estado, su estructura, su capacidad de gestión, su régimen de administración pública, la formación de sus agentes y en general el funcionamiento de sus instituciones (públicas y de la sociedad civil que participan en los procesos de decisión gubernamental) son condicionantes para responder no solo ante crisis como la actual, sino también para la elaboración de estrategias, negociaciones de consensos, acuerdos, hojas de ruta para el mediano y largo plazo.

Existe abundante literatura sobre la tesis por la cual "las instituciones cuentan" y que el funcionamiento de la economía y de la sociedad en su conjunto puede medirse en términos de sus "costos de transacción institucionales", medidos en ciclos históricos (North, 1990).Por su parte, Daron Acemoglu y James Robinson, (2012), simplifican las categorías de los estados en dos: "estado extractivo vs estado inclusivo" en función de la historia de los países medidos a lo largo de todas sus historias. El estado extractivo es básicamente un estado que solo extrae los recursos de sus habitantes y que a la vez, establece mecanismos que impiden su crecimiento y desarrollo económico y social. Por su parte, el estado inclusivo es el que garantiza la seguridad jurídica, el derecho de propiedad y los derechos individuales, a la vez que cuenta con 
instituciones que permiten la generación de oportunidades para el desarrollo económico y social de sus habitantes.

Obviamente, estos análisis de tipo general no explican por sí solos el atraso del "País de las desmesuras: raíces del retraso de la Argentina" (Llach y Lagos, 2016) ni tampoco pueden entenderse las "instituciones" como un bloque según Eduardo Levy Yeyati (2021), como único causante del retraso argentino. Pero lo que sí es cierto es que si las existentes no se adaptan, mejoran y en algunos casos se construyen nuevas y se eliminan otras, cualquier camino que pretenda llevar a la construcción de una Argentina basada en el sentido común y con una adecuada vinculación con el mundo exterior que le permita contribuir a superar su atraso, será infructuoso. $\mathrm{Y}$ es en este punto donde la diplomacia cuenta como un instrumento clave para contribuir conceptualmente y operativamente en la elaboración de una estrategia para contrarrestar el desarrollo perdido.

Vayamos a la evidencia de lo que se intenta demostrar según los datos del Informe sobre el Desarrollo Humano del PNUD (informes anuales desde el 1990 al 2020) que incluye varios indicadores sobre el desarrollo humano. EL IDH (Índice sobre Desarrollo Humano) general ubicaba a la Argentina en el año 1989 en el 34 lugar en la categoría de desarrollo humano alto. El último informe ubica al país en el puesto 48. El punto a destacar no es solo la caída en el ranking, sino que otros países de la región -los cuales en todo caso, están afectados por las mismas tendencias que la Argentina-, han mejorado su ubicación, obviamente mejorando la calidad de vida de sus habitantes, en los casi 50 indicadores que incluye la elaboración de ese índice.

Sucede lo mismo con los indicadores de competitividad global (posición 83 en el 2019) y de transparencia institucional (posición 66 en el 2019). Ambos basados en indicadores del año 2018 para 180 países.

Revertir esta tendencia al atraso depende de políticas públicas domésticas, pero también el cuerpo diplomático, actuando como puente hacia ambos lados con el mundo exterior, puede contribuir a proyectar a la Argentina - como parte del MERCOSUR- como un país viable. Pero ello deberá ser a partir de contar con seguridad jurídica, instituciones confiables, respeto a las reglas de juego y a los derechos de propiedad, sistemas impositivos y laborales simplificados, capacitados y basados en la capacidad de generar empleo y sobre todo orientados hacia la industria del conocimiento que haga a la incorporación, disponibilidad y generación de tecnologías para la mejora de su competitividad. De esta manera se podrá garantizar inversiones (externas y asociadas al capital local), participación en los mercados financieros internacionales, promover la participación de empresas argentinas en las Bolsas de Comercio con acceso a los mercados de capitales, incentivar el comercio internacional hacia ambos lados basado en la complementariedad económica y que a su vez estos flujos comerciales sean promotores de inversión.

Sin duda alguna, actividades en sectores -entre otros- como: los vínculos interparlamentarios, la educación, el conocimiento aplicado, la academia, la defensa, el desarrollo social en sus múltiples sectores, el enfoque de los temas de soberanía en relación a la integridad territorial, la aplicación de las convenciones vinculantes en materia de derechos humanos, la cooperación judicial, la cooperación para la lucha contra la corrupción, el narcotráfico y la trata de personas, las cuestiones migratorias, las políticas de no proliferación, el medio ambiente y desarrollo sustentable, las artes, el turismo, el deporte y como tantas otras que a través que puedan identificarse en función de la hoja de ruta que la Argentina elabore, también serán claves para su vinculación con el mundo exterior.

En el actual contexto internacional post COVID-19 van a predominar los acuerdos específicos entre países y/o agrupaciones regionales por encima de las normas internacionales que hoy regulan el comercio y las inversiones. Si bien, el multilateralismo ha sido, es y debiera ser el mejor escenario para la Argentina por su relativa capacidad (tendencia decreciente) de incidir en las decisiones de los temas globales, el futuro próximo indica que la 
Argentina/MERCOSUR deberá pensar en estrategias alternativas para su relación con el mundo exterior. La OMC ha demostrado que no tiene capacidad para que se apliquen sus normas en materia de comercio exterior. En el actual contexto predominan los Acuerdos de Libre Comercio (que son en realidad de "facilitación de comercio" y van mucho más allá de los sistemas de preferencias comerciales) entre países y organizaciones regionales.

Por otra parte, la transnacionalización del comercio exterior hace que los flujos comerciales y financieros se basen en acuerdos entre empresas globales. En el caso de las organizaciones multilaterales de crédito si bien son influyentes, no cuentan con la capacidad de inyectar liquidez ante las crisis financieras ni de financiar los proyectos de infraestructura que contribuyan a mejorar la competitividad de los países miembros. Por su parte, el conjunto de las instituciones de la familia del sistema de las Naciones Unidas, quizás con las excepciones del Programa Mundial de Alimentos (PMA) y del Alto Comisionado de las Naciones Unidas para los Refugiados (ACNUR) que hacen frente a las crecientes demandas de asistencia humanitaria con gran eficiencia y eficacia, no dan muestra de dar soluciones concretas a las cuestiones de fondo de la agenda global, a pesar de las bien intencionadas políticas multilaterales que los países miembros adoptan en cada una de ellas. La cooperación al desarrollo bilateral y multilateral acompaña estas tendencias.

Los mecanismos de integración regionales y subregionales, así como los países, tratan -cada vez más- de encontrar soluciones a sus propias problemáticas, en un contexto donde el estado-nación prevalece cada vez más. Las disputas comerciales entre las dos principales potencias económicas y las disputas geopolíticas de potencias secundarias con aspiraciones de liderazgos regionales (y en algunos casos globales) generan climas de inestabilidad y presión en materia de alianzas basadas en la geopolítica. Asimismo, las distintas problemáticas específicas, entre otras, medio ambiente, migraciones, refugiados, terrorismo, cyber-terrorismo, dominio de los mares y el espacio, dependen cada vez mas de los países que de los actuales o eventuales acuerdos multilaterales.

En un mundo ideal, no debiera ser así porque todos estos temas requieren de la cooperación internacional. Pero la realidad indica que éstos son los escenarios a tener en cuenta al momento de elaborar una estrategia de inserción multi y bilateral, ya sea a nivel de Estados como de vinculación con las organizaciones de la sociedad civil que cada vez más influyen en las decisiones de políticas de los gobiernos. La no comprensión de estos escenarios internacionales por el gobierno argentino, será -sin duda alguna- un impedimento para una política doméstica de crecimiento económico sostenido y desarrollo sustentable que permita generar las asociaciones y alianzas diversas según los intereses estratégicos del país. Hete aquí otro valor agregado que la diplomacia cuenta.

Los niveles de competitividad y de transparencia institucional que el país pueda demostrar, no serán solo las únicas cuestiones -como debiera ser de todas maneras- que hacen a un estado inclusivo para que sus más variadas instituciones funcionen en forma acorde, pero sí es cierto que serán cruciales para ser un país atractivo (más allá de sus bellezas naturales, la calidad de su gente y de su tan mencionado "potencial") para integrarse cada vez más a un mundo complejo y cambiante. Y, es en el contexto arriba descripto, donde el estado argentino (priorizando donde el mandato lo permita como MERCOSUR), a través de su política exterior entre otras- debe: facilitar y negociar las alianzas internacionales con estados y agrupaciones regionales en función de sus intereses estratégicos, modelo de país y complementariedad económica ; participar en las organizaciones intergubernamentales; estimular a que su sector privado promueva acuerdos asociativos favoreciendo las cadenas de proveedores a nivel internacional; promover el valor agregado en su producción (sin diferenciación, este dirigida al mercado interno o externo ya que los estándares internacionales primaran cada vez más en la producción de bienes y servicios); impulsar asociaciones y distintos tipos de colaboración a nivel privado (ejemplo en matera de certificaciones) y entre organizaciones de la sociedad civil que contribuyan a erradicar las limitaciones que impiden al país sus vínculos sectoriales con los estados y con las instituciones privadas (a título de ejemplo, reconocimiento de títulos y 
equivalencias en las universidades públicas). De ser así, se iniciará un largo camino que mejorará - en forma definitiva- los indicadores argentinos en materia de competitividad y transparencia institucional.

En términos concretos, si se cuenta con el diagnóstico correcto, la política exterior argentina, instrumentada por su cuerpo profesional, -además de sus actividades de rutina-, poniendo el foco en la identificación de oportunidades para generar los instrumentos internos y externos que diseñen, faciliten y ejecuten su estrategia de vinculación global, seguramente podrá contribuir, en el actual contexto internacional, a reducir sus niveles de pobreza, mejorar la calidad de vida de su pueblo, su calidad institucional y a la vez reflejar en el ámbito externo sus valores, su cultura, sus costumbres y su compromiso con solución de los temas identificados por la agenda global.

Síntesis: la política exterior comienza por casa, e instrumentada por su cuerpo profesional permanente será clave para contribuir a la solución del atraso argentino.

\section{Bibliografía}

Acemoglu, D.y Robinson, J. (2012), Why Nations fail? The origins of power, prosperity, and poverty, Nueva York, Estados Unidos: Crown Publishing Group

Haass, R.N, (2013), Foreign Policy Begins at Home: The Case for Putting America's House in Order, Nueva York, Estados Unidos: Basic Books

Llach, J.J y Lagos, M. (2016), País de las desmesuras: raíces del retraso de la Argentina, Argentina, El Ateneo

North, D.C (1990), Instituciones, cambio institucional y desempeño económico, D.F, México, Fondo de cultura Económica

Sersale, C. (2002), La Declinación Argentina, Archivos del Presente, Debates, Revista Nro 27, pp.7, recuperado de: https://archivosdelpresente.com/articulos/la-declinacion-argentina/? sf s $\mathrm{s}=$ Debate + sobre + la + declinaci $\% \mathrm{C} 3 \% \mathrm{~B} 3 \mathrm{n}+$ argentina

Yeyati, L. E, (2021), Dinosaurios y Marmotas: en busca del desarrollo perdido, Argentina, Capital Intelectual

TRABAJO RECIBIDO: 27/10/2021 